\title{
Intelligent computational control of multi-fingered dexterous robotic hand
}

\author{
Disi Chen ${ }^{1}$, Gongfa $\mathrm{Li}^{1^{*}}$, Guozhang Jiang ${ }^{1}$, Yinfeng Fang ${ }^{2}$, Zhaojie $\mathrm{Ju}^{2}$ and Honghai Liu ${ }^{2}$ \\ ${ }^{1}$ College of Machinery and Automation, Wuhan University of Science and Technology, Wuhan 430081, China \\ ${ }^{2}$ Intelligent Systems and Biomedical Robotics Group, School of Computing, University of Portsmouth, Portsmouth, PO1 3HE
}

\begin{abstract}
We discuss the intelligent computational control theory and introduce the hardware structure of HIT/DLR II dexterous robotic hand, which is the typical dexterous robotic hand. We show that how DSP or FPGA controller can be used in the dexterous robotic hand. A popular intelligent dexterous robotic hand control system, which named Electromyography (EMG) control is investigated. We introduced some mathematical algorithms in EMG controlling, such as Gauss mixture model (GMM), artificial neural network (ANN) and Support vector machine (SVM).
\end{abstract}

Keywords: Dexterous Hand, Intelligent Control, EMG Control.

\section{INTRODUCTION}

Robot, as a technological representative in the new era, becomes the industry hot spot of both information technology and traditional mechanical industry. These automatic devices, which could function like our human beings, can not only replace human beings in some production aspects to improve the productivity greatly ${ }^{1}$, and reduce the cost pressure of mankind labor, but also simulate human's thinking or action pattern to complete some higher flexible activities.

A report, named "Robots Moving Closer to Humans", was given by Professor Siciliano ${ }^{2}$ on the 11th International Conference on Control, Automation, Robotics and Vision (ICARCV2010), he proposed that the robot was getting closer and closer to people, and they could interact, study and work with human beings. Most of robotic hands, as the end effector ${ }^{3}$ for robot to interact with outer space, used to finish tasks following the guidance of program codes Their capability had been greatly enriched with the support of information technology, but the structure of most robotic hands are still very simple, such as clamp, pliers and so on. They were designed for certain kind task. As for complex tasks, it was difficult to take all circumstances into consideration. Multi-fingered dexterous robotic hand was designed to solve these problems by using the bionic structure, the multi-fingered hand become more flexible and more similar to human hand. Using the intelligent control technology largely reduced the dependence of programming, and greatly improved the adaptability and learning ability of dexterous robotic hand. Dexterous robotic hand should also have the similar capability as human hand do, such as enough position accuracy in grasping operation, and precise force controlling in grasping fragile objects, which requires the dexterous robotic hand to be equipped with multiple sensors ${ }^{4}$ (such as position, force, temperature detection, and the sensation of touch and slip, etc.). Due to the uncertainty of the working environment, the anti-interference ability was important for dexterous robotic hand. These problems were inevitable in designing of dexterous hand control system.

\section{EMBEDDED CONTROL SYSTEMS OF DEXTEROUS ROBOTIC HAND}

Intelligent control was an interdisciplinary, as K. S. Fu claimed, the intelligent control was an interdisciplinary of the artificial intelligence and automatic control. After that, G. N. Saridis ${ }^{6}$ added operational research into intelligent control, and he held the view that intelligent control was the intersection of artificial intelligence, operational research and automatic control, as shown in figure 1. 


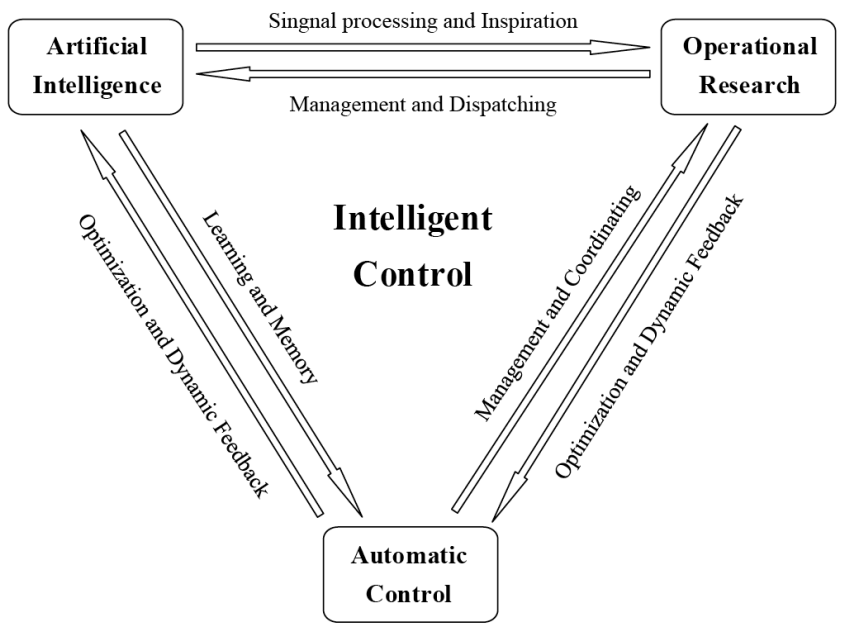

Fig. 1. Intelligent control

Intelligent control system was actually the further development of the traditional control system ${ }^{7}$, which should be able to adapt to different settings, organize different tasks and had the function of automatic learning. The self-adaptive learning control system was a much more universal control system compared to self-adaptive or self-organizing control system. It could not only be used to control the system without knowing its dynamic characteristics, but also could be used as a superior decision pattern recognizer, classifier or manager. A control system, which contained those features above could be regarded as learning control system. The learning control system designed for robotic hand controlling $^{8}$ had the ability to learn information of intrinsic property from an unknown process or environment, and could use the information learned from that to control one process with few unknown variables. Pattern classification, Bias estimates, Stochastic approximation and Fuzzy automata model were the mathematic methods most widely used ${ }^{9}$ in research learning control systems.

\subsection{Intelligent control system of multi-fingered hand based on DSP/FPGA}

HIT/DLR II dexterous hand was designed by the Harbin Institute of Technology (HIT) and the German Aerospace Center (DLR) ${ }^{10}$, with 4 fingers sharing the same structure and one independent thumb. The total degrees of freedom of the hand was 13, and there were many sensors for testing position, force, torque and temperature. Compared with HIT/DLR I, the previous generation HIT/DLR hand, HIT/DLR II contained five fingers rather than four fingers on HIT/DLR $\mathrm{I}^{11}$, and its structure was more compact to match the shape and size of human hand.

\subsection{General structure of the control system of dexterous hand}

The control system of DLR/HIT II hand was designed on the basis of DSP/FPGA control structure ${ }^{12}$, according to the hierarchical controlling and modularization theory. The sensing system, driving control system and the machine body of the hand were intelligently integrated together by using electronic components and the high efficient controlling constructions, as shown in figure 2 . All data processing and control algorithm were divided into 5 layers. The bottom data collection, processing and electric motor driving were controlled by the DSP and FPGA sensors in fingers. However, in the communication layer, all the signal were transferred by FPGA in the palm to control the movement of each finger. In the higher control layer, task planning and multi-finger manipulating were organized by floating point $\mathrm{DSP}^{13}$, and the clock cycle of the whole control system was 200 s. Finally, all the man-machine interface on top layer was supported by PC.

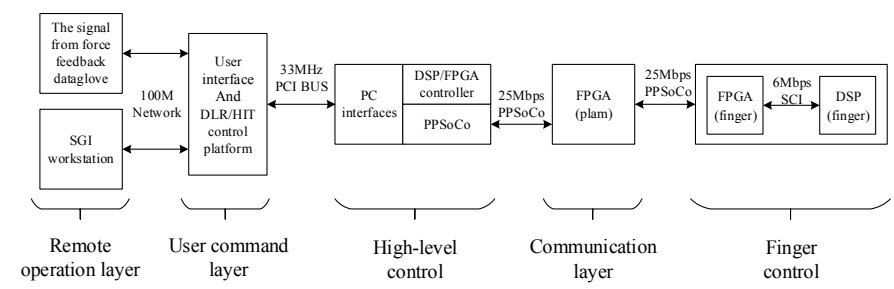

Fig. 2. Layer structure of the control system of DLR/HIT II hand

\subsection{System structure of the finger control layer}

DSP controller ${ }^{14}$ in the tip of the HIT/DLR II finger was in charge of collecting and processing data which were sent by sensors on this finger. The control of joint and communication between FPGA in finger were all controlled by DSP, as shown in figure 3 .

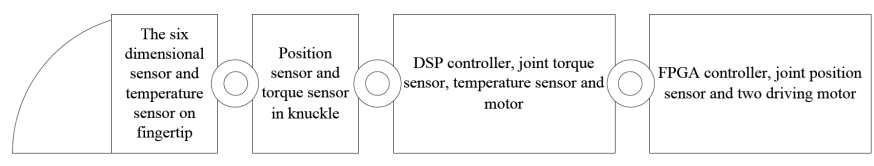

Fig.3. Internal structure of HIT/DLR II finger

DSP in the knuckle was able to execute millions of instructions per second, which was important for fast computation. Moreover, there were abundant peripheral resources supported by this chip, such as enhanced pulse width modulation (PWM) module for motor drive, capture (CAP) module for digital Hall signal cap-turing ${ }^{15}$ from motor, analog to digital conversion (A/C) module for sensor signal collection, serial peripheral interface (SPI) module for serial communication with the external $\mathrm{AD}$ sensor and serial communication interface (SCI) module for the communication with FPGA $^{16}$ in fingers, as shown in figure 4 . 


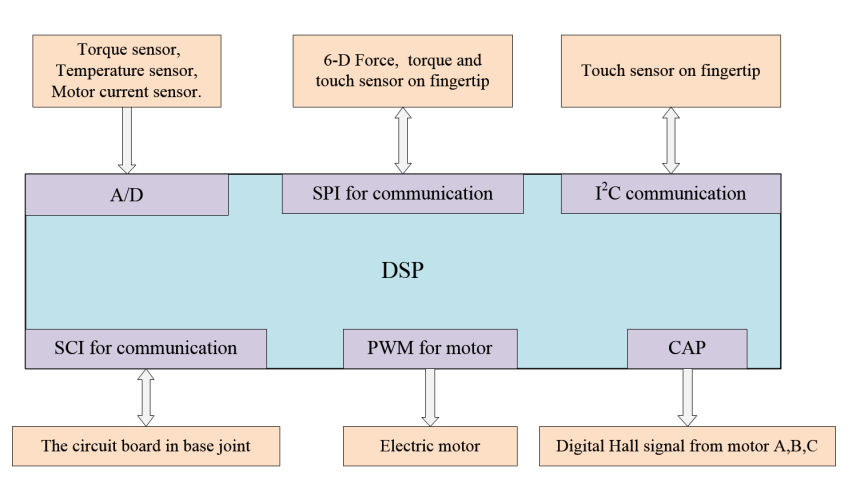

Fig. 4. DSP control system in finger

FPGA controller in base joint of a finger was in charge of the sensors' signal calibration, driving the base joint and the communication between palm FPGA and finger DSP. The finger FPGA was embedded with 32 bit RISC microprocessor $^{17}$ (NIOS), and used the hardware description language (VHDL) to run other program module, such as serial communication interface, serial peripheral interface and driving the brushless DC motor ${ }^{18}$, as shown in figure 5. Since digital signals collected by sensors contained no physical meaning, they could not be directly applied in the control algorithm ${ }^{19}$. The collected signals, after calibrating, should be converse into useful information by finger FPGA, such as position, speed, torque and temperature ${ }^{20}$ etc. Then the useful information would be packed to send to the upper controller.

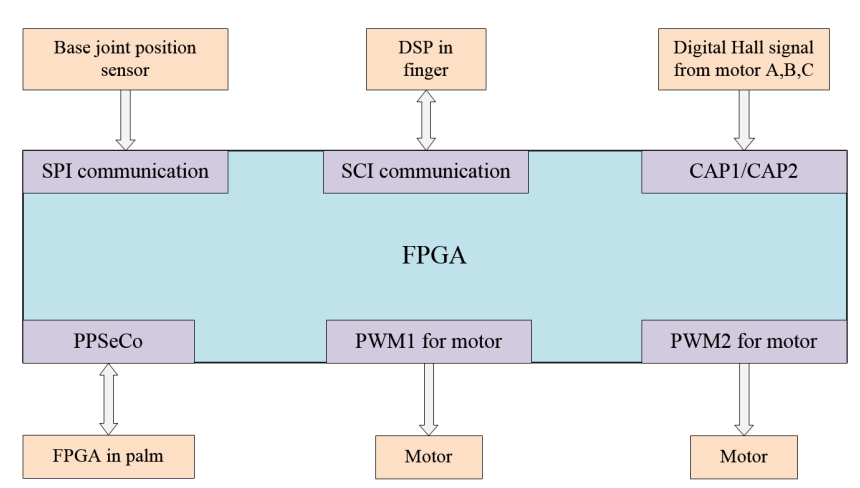

Fig. 5. FPGA control system in finger

\section{EMG PATTERN RECOGNITION BASED CONTROL SYSTEM OF DEXTEROUS HAND}

The EMG control of robotic hand ${ }^{21}$ was a typical human-computer system. There were three main purposes of human-computer interaction. The first one was to regard human as a part of the system, and tried to study the behavior of the whole system. The second one was to simulate the human characteristics in system, so as to realize the human kind intelligent control in further. The third one was to study the characteristic of human and computer respectively, and to combine the decisionmaking ability of human and the quick response ability of computer, in order to take good advantage of them, and to construct a human-computer intelligent control system effectively. The EMG control system of dexterous robotic hand belonged to decision-making model ${ }^{22}$ control system in advance, and man held the dominant position. Because different people had different muscle and nerve structure, in the construction of the EMG signal system, it was necessary to take different participators in the system validation. Even so, some researchers pointed out that the EMG control system was not universal valid ${ }^{23}$, the validity of the system only need to be tested on individual participator. The EMG signal of human body was the original source of control signal, so the quality ${ }^{24}$ of EMG signals was essential to the accuracy and reliability of the robotic hand operation. The long term effective EMG output training based on different modes of was a method to improve the success rate of EMG control. To set up the suitable EMG control system, the mechanism of EMG output training $^{25}$, and the structure of nerve and muscular on arm and should be studied.

\subsection{Structure of EMG control system}

The EMG control not only related to the theory of automatic control, but also related to the biological information processing theory. EMG control process was based on the pattern recognition algorithm. As shown in Figure 6, the EMG feature extraction and pattern recognition were needed in EMG controlling, which belonged to the flied of computer pattern recognition. The performance of EMG control system was determined by recognition system, which was the core of EMG control system. Because there were no accurate mathematical model of EMG pattern recognition at present ${ }^{26}$, the only way was conducting experiments and trying different pattern recognition method representative to pick out the experiential optimal extractor and classifier ${ }^{27}$.

After the EMG pattern of a certain action or posture was obtained, EMG signal need to be transmitted to the controller of dexterous robotic hand. Then the signal would be translated into motion parameters for the input of motion trajectory planning by the motor controller. In controlling the grasping mode of robotic hand, force information also needed to be extracted from EMG signals, so that the controller could distribute grasping force in each finger. Dexterous robotic hand also required position, joint torque sensors for propose of learning the current position and force information of each finger. In addition, to get the man know about the current status information, vibration feedback and electrical stimulation 28 were most used as the feedback methods, the hierarchical feedback was structure of force feedback. However, the location information of the prosthetic hand was still rely on the visual feedback. How to make the robot feedback information recognized from the outside world to human body, and follow the normal nerve transmission pathways in the human body, was a big problem in EMG control, it also played a pretty important role in the closed-loop EMG control system. 
Fig. 6. Structure of FPGA control system in fingers

A typical EMG control system should include the EMG signal output from a body, biological information acquisition and processing, action pattern recognition, robotic hand controlling and biological feedback ${ }^{29}$. Those five basic components above were shown in figure 7 . The transfer of all kinds of information between the different levels could be summarized that the impulse from the central nerve of human body was transferred to skeletal muscle and nerve interface via nerves. The impulse was amplified by the motor endplates to outbreak the ${ }^{30}$ action potentials. All action potentials were overlapping to cross the tissue fluid, fat and skin to reach the surface. The electrode on the surface would collect and pre-process the EMG signal from human body. The EMG signal which stood for hand muscle contraction mode was recognized in different patterns to obtain the instruction of robotic hand movement. Finally, the internal control loop was formed with its own sensors to, and the interaction between internal and external of the robotic hand were in the form of feedback (vision, electrical stimulation and vibration) to transmit to the human body, which was the closed loop within human body ${ }^{31}$.

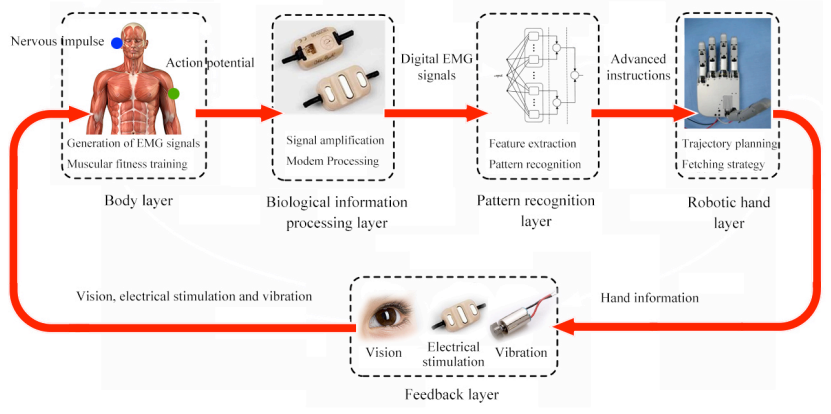

Fig. 7. Structure of EMG control system

\subsection{Mathematical mechanism of EMG signals}

The EMG signal $x(t)$ collected by each electrode was the sum of $M$ motor unit action potentials(MUAP) :

$$
X(t)=\sum_{i=1}^{M} m_{i}^{(t)}
$$

According to the theory of electrophysiology, when muscle contraction force, changed, neuron pulse frequency was almost the same. However, the total number of MUAP which defined as $M$, was turning into $M(t)$, a time-varying function. The EMG signal is:

$$
x(t)=c(t) \sum_{i=1}^{M} m_{i}^{(t)}
$$

To reduce the interference of muscle electricity, human body electrostatic field and the power frequency of circuit, improve the common-mode rejection ability of EMG signal, two same electrodes were used along the axis direction of the muscle fibers, with the distance of $10 \mathrm{~mm}$ between two EMG signal collecting positions. The signal taken was differential signal:

$$
\mathrm{z}(t)=\mathrm{x}(t)-x(t-\Delta t)
$$

Where, $\Delta t=d / v, d$ was the electrode distance, $v$ was the electrical conduction velocity, $\Delta t$ was the delay of action potential from the first electrode to the second electrode. As a result, the power spectrum of EMG:

$$
\begin{aligned}
S_{\mathrm{z}}(\omega) & =S(\omega)(1-\cos (\omega \Delta t)) \\
& =4 S_{x} \sin ^{2}(0.5 \omega \Delta t)
\end{aligned}
$$

From formula (1), double electrode collected EMG signal $z(t)$ was the differential of $x(t)$, in the integer times of $1 / \Delta t$ of $z(t)$, power spectrum subsided, namely when $S_{\mathrm{x}}(\omega)=0$ in formula (2), the EMG signal double electrode was typically non-stationary signal or timefrequency domain signal and the spectrum feature weight was larger than the amplitude. Therefore, the series of DB wavelet packet transform, (WPT), from the time domain signal $z(t)$ to extract the frequency domain information $z(f)$. Each signal is WPT is decomposed into eight frequency band:

$$
\left(\left(s_{i, 1}^{1,2, \cdots, j}, \cdots, s_{i, t}^{1,2, \cdots, j}\right), 1, \cdots,\left(s_{i, 1}^{1,2, \cdots, j}, \cdots, S_{i, t}^{1,2, \cdots, j}\right), 8\right)
$$

and their energy were:

$$
E_{k}=\frac{1}{N_{s}} \sum_{i=1}^{N_{s}}\left|S_{k}(i)\right|
$$

It was WPT refactoring coefficient module, among them, $k=1,2, \ldots, 8, s_{k}$ was the number $k$ WPT sub-band coefficient of refactoring, $1,2, \ldots, j$ was the number of muscles EMG and $N_{s}$ was the rebuild coefficient of WPT, its value was equal to the number of sampling time $t$. Thus, each electromyography value of action sample were $\left(x_{1}, x_{2}, \cdots, x_{8}\right)$, among them $x_{k}=E_{k}(s)$.

\subsection{Popular pattern recognition algorithms in EMG control}

\subsubsection{Linear classifier}

Linear classifier (LLC) ${ }^{32}$ was a linear function of the input vector $\mathrm{x}$, which was defined as follows:

$$
g(x)=W^{T} x+b
$$

Where $\boldsymbol{w}$ was a weight matrix for linear transformation, $b$ was a classifier bias. Linear classifier decision boundary was a linear hyper plane. In a classification problem of two class, given a feature vector $\boldsymbol{x}$, if $g(\boldsymbol{x})>0, \boldsymbol{x}$ was marked as $\omega_{1}$, otherwise, the $\boldsymbol{x}$ would be marked as $\omega_{2}$. In this paper, we used a logarithmic multi-class linear 
classifier. In a $N C$ problem, the linear classifier was defined as:

$$
\log \left(\frac{p\left(x \mid \omega_{i}\right)}{p\left(x \mid \omega_{N C}\right)}\right)=\beta_{i 0}+\beta_{i}^{t} x, \quad i=1,2, \ldots, N C-1
$$

The parameter of linear classifier, such as $\beta_{i 0}, \beta_{i}, i=1$, $2, \ldots, N C$ could be estimated by the maximum likelihood $\operatorname{method}(\mathrm{ML})$.

\subsubsection{Gauss mixture model classifier}

Gauss mixture model (GMM) classifier was a classification method based on probability model, which has been widely used in voice recognition ${ }^{33}$. Because there are lots of similarities of EMG and voice signals, the GMM classifier had been applied to the EMG pattern classifier successfully, and identified 6 kinds of wrist and hand movements ${ }^{34}$. For a $N C$ classification problem, when a $\mathrm{D}$ dimensional input feature $\boldsymbol{x}$ was given, the type $i$ GMM hybrid likelihood function was:

$$
p\left(x \mid \omega^{i}\right)=\sum_{r=1}^{M} \lambda_{r}^{i} p_{r}^{i}(x), \quad i=1,2, \ldots, N C
$$

Where $M$ was the number of mixing elements of Gauss, $\lambda_{r}^{i}, r=1,2, \ldots, M$ were the mixing coefficient, which met the following constraint conditions $\sum_{r=1}^{M} \lambda_{r}^{i}=1$, $\lambda_{r}^{i} \geq 0$, the density function of each hybrid element $p_{r}^{i}(x), r=1,2, \ldots, M$ was a single mode probability distribution function:

$$
p_{r}^{i}(x)=\frac{1}{(2 \pi)^{D / 2}\left|\sum{ }_{r}^{i}\right|^{1 / 2}} \exp \left\{-\frac{1}{2}\left(x-\mu_{r}^{i}\right)^{T}\left(\sum_{r}^{i}\right)^{-1}\left(x-\mu_{r}^{i}\right)\right\}
$$

Where $\boldsymbol{\mu}_{r}^{i}$ was the mean vector of a $\mathrm{D}^{*} 1$ dimension, and the covariance matrix was $\mathrm{D}^{*} \mathrm{D}$ dimension.

The above definition showed that the GMM model was completely determined by $\omega_{i}=\left\{\lambda_{i}^{r} \mu_{i}^{r} \sum_{i}^{r}\right\}, r=1,2, \ldots$, $M, i=1,2, \ldots, N C$. These parameters should be accurately estimated in the training process of GMM classifier. Now the popular method to estimate the parameters of GMM classifier was the expectation maximization algorithm (EM).

\subsubsection{Artificial neural network classifier}

Artificial neural network (ANN) was another popular mathematic tool, which was able to achieve the da-ta classification, regression and other functions. The most simple network structure was a two-layer perceptron network. It could be regarded as a linear classifier. The most significant drawback of two-layer network was it could only deal with linear pattern recognition problem. With the introducing of multi-layer perceptron neural network, it was able to handle nonlinear problems, which greatly expanded the application scope of neural network. In this paper, a three-layer neural network classifier was chosen, for the three-layer network can approximately deal with any complex system in theory. In the EMG classifier, this kind of sensor network was also been used. The definition of three-layer artificial neural network classifier was shown as follows:

$$
y_{k}(x)=h\left(\sum_{j=0}^{M} W_{k j}^{(2)} \cdot \sigma\left(\sum_{1=0}^{D} W_{j i}^{(1)} X\right)\right), k=1,2, \ldots, N C
$$

Where, $\boldsymbol{x}$ was the input vector, the output value was $\boldsymbol{y}$. $W_{j i}^{(1)}, W_{j i}^{(2)}$ were the moment of the first layer and the second layer. $\sigma(\bullet), \mathrm{h}(\bullet)$ were the activation function of neurons. In pattern recognition, $\sigma(\bullet)_{\text {stood as a sigmoid }}$ function:

$$
\sigma(\mathrm{x})=1 /(1+\exp (\mathrm{x}))
$$

$h(\bullet)$ was a linear function, its range is fixed in the interval $[0,1]$, the error back propagation algorithm was commonly used to complete the training of MLP. In order to enhance robustness generalization ability of the network $^{35}$, the regularization technique was used to prevent over large of some weights in the network, which might cause the over fitting phenomenon.

\subsubsection{Support vector machine classifier}

Support vector machine (SVM) classifier, a new kind tool of machine learning, was proposed recently, which has been widely used now. Unlike other methods to minimize the error or error rate, the goal of SVM was to maximize the edge of different classes. There were some features of SVM as follow:

(1) By using the kernel function ${ }^{36}$ to map the input vector into a high-dimensional space, in order to obtain better separation properties.

(2) According to the computational statistical theory ${ }^{37}$, the distance from one class to the discriminant hyper plane would be maximized.

The basic structure of the SVM classifier was a twoclass linear classifier problem, the expression was $y=w \cdot \phi(x)+b$, where $w$ was the weight matrix of classifier, $\boldsymbol{b}$ was the offset value matrix, and $\varnothing(x)$ was the kernel function, the kernel function was defined:

$$
k\left(x, x^{\prime}\right)=\phi(x)^{T} \phi\left(x^{\prime}\right)
$$

Through the appropriate selection of the kernel function, the input vector could be implicitly mapped into a high dimensional space ${ }^{38}$, without direct operation in high dimensional space, which was called kernel tricky. In order to make the SVM become nonlinear, we used 
radial basis function $^{39}$ as the kernel function, because it was a basic function in pattern recognition problem. In samples, the target values of the tag was set as $t_{i} \in\{-1,1\}$, all samples meet the following conditions:

$$
t_{n}\left(W^{T} \cdot \phi\left(x_{i}\right)+b\right) \geq 1, \quad i=1,2, \ldots, N
$$

When the sample points located in the super plane, the equality holds and it was called support vector. In practice, there would be overlap between classes, so we need to introduce the slack variables $\xi_{i} \geq 0$. In solving the softmargins SVM problem ${ }^{40}$, the optimized expressions were as follows.

$$
\underset{W, b}{\arg \min } \frac{1}{2}|W|^{2}+C \sum_{i=1}^{N} \xi_{i}
$$

Where $C \geq 0$, it represents the punishment coefficients of a slack variable. Its role was to compromise the margin maximization and error minimization problems ${ }^{41}$. The final solution to the optimization problem was a dual Lagrange equation:

$$
\tilde{L}(\alpha)=\sum_{i=1}^{N} \alpha_{i}-\frac{1}{2} \sum_{i=1}^{N} \sum_{j=1}^{N} \alpha_{i} \alpha_{j} t_{i} t_{j} k\left(x_{i}, x_{j}\right)
$$

In the EMG classification, we need to extend the SVM classifier from two-class classifier to handle multi-class recognition problems. Researchers have proposed many methods to solve this problem, such as one against rest (OAR), one against one (OAO), directed acyclic graph (DAG), the two fork tree, etc. Research showed that OAO and $\mathrm{DAG}^{42}$ was the optimized method compared with others in actual calculation.

\section{CONCLUSIONS}

In this paper, the HIT/DLR II dexterous hand was taken as an example, to introduce the main driving mechanism, sensors and other hardware of a dexterous hand. Then, introduced the EMG control strategies of dexterous hand in detail, and summarized the application of mathematical theory of intelligent control in the EMG control of dexterous hand. Intelligent control was an important part in robotic hand motion, with the development of computer technology, it had been more and more intelligent. At the same time, the robotic hand was becoming more flexible, and was able to work seamlessly with the human under the control of intelligent control system. The use of EMG control made it possible for robot to act as a prosthetic. In the near future, with the emerging of new computational control algorithm, the robotic hand will continue to upgrade, to obtain the even higher precision and power than human hand.
Acknowledgments: This research was supported by grants of National Natural Science Foundation of China (Grant No.51575407, 51575338, 51575412) and the UK Engineering and Physical Science Research Council (Grant No. EP/G041377/1)

\section{References and Notes}

1. J. Yu, Manufacturing Technology \& Machine Tool, 21 (2013).

2. M. K. Al-Azzawi, J. Luo, R. Li, and J. Waleed, J. Comput. Theor. Nanosci. 12, 316 (2015).

3. X. Wang and G. Shi, Robot. 31, 493 (2009).

4. J. Liu, Y. Zhang and B. Liu, Robot, 25, 444 (2003).

5. K. Qian, A. Song and H. Zhang, Journal of Southeast University (Natural Science Edition), 42, 637 (2012).

6. G. Chen, W. Zhang, Z. Gong, W. Sun and M. Zhao, Chinese Journal of Scientific Instrument, 30, 1836 (2009).

7. X. Wang, R. Zhang, G. Gu, Journal of Computer Research and Development, 40, 1444 (2000).

8. G. Xie, X. Liang, H. Minoru and X. Zhuang, Control Theory \& Applications, 26, 589 (2009).

9. C. Wei, Y. Zhao and H. Wang, Journal of Mechanical Engineering, 47, 43 (2011).

10. T. Lan. Y. Liu, M. Jin, L. Jiang, S. Fan and H. Liu, Electric Machines and Control, 13, 608 (2013).

11. L. Hong, P. Meusel and Seitz, Mechanism and Machine Theory, 42, 612 (2007).

12. G. Singh Patel and K. Sharma, J. Comput. Theor. Nanosci. 10, $2458(2013)$

13. C. Wei, Y. Zhao, H. Wang. Journal of Mechanical Engineering, 47, 43 (2011).

14. X. Yang and G. Zhou, X. Chen, Journal of Central South University of Technology, 32, 428 (2001).

15. Y. Huang, Q. Liao, S. Wei and L. Guo, Computer Measurement \& Control, 19, 2965 (2011).

17. T. Lan, Y. Liu, M. Jin, L. Jiang, S. Fan and H. Liu, Electric Machines and Control, 4, 608 (2009).

18. Y. Ao, Journal of Guangdong University of Technology, 21, 5 (2004).

19. X. Chen, M. Tan, T. Ye and L. Li, International Electronic Elements, 9, 4 (2002).

20. J. Li, S. Dong and L. Hong, Study on Inverse Kinematics and Trajectory Tracking Control of Humanoid Robot Finger with Nonlinearly Coupled Joints, Proceedings of the 2007 IEEE International Conference on Mechatronics and Automation, (2007) 3214-3219; Harbin, China.

21. H. Chai, C. Shi, H. Wang, K. Zhang, K. Yang, R. Zhao and X. Zhang, Chinese Medical Equipment Journal, 34, 81(2013).

22. G. Xu, A. Song and H. Li, Journal of Southeast University (Natural Science Edition), 39, 156 (2009).

23. Y. Fang, H. Liu, G. Li and X. Zhu, International Journal of Humanoid Robotics, 12, 1 (2015).

24. D. Yang, J. Zhao, L. Jiang, R. Liu and H. Liu, Machinery \& Electronics, 12, 7 (2009).

25. L. Zhang, Y. Su, W. Yang, G. Li, International Journal of 
Biomedical Engineering, 36, 311 (2013).

26. D. Yang, J. Zhao, N. Li, L. Jiang and H. Liu, Journal of Mechanical Engineering, 48, 1(2012).

27. T. Lu, S. Zhang, Chinese Journal of Physical Medicine and Rehabilitation, 25, 169 (2003).

28. D. Yang, Research on Recognition System of Multi-Channel EEG Based on Specific Mental Tasks Symposium on Assembly and Task Planning, Harbin Institute of Technology, (2006).

29. Q. Tang, Development of an Embedded Controller for Prosthetic Hand Based on Speech and EMG Signals, Harbin Institute of Technology, (2008).

30. Y. Yang, X. Chen, Y. Tu, X. Zhang and J. Yang, Journal of System Simulation, 22, 651 (2010).

31. Y. Li, X. Chen, X. Zhang, Z. Zhao and J. Yang, Space Medicine \& Medical Engineering, 23, 419 (2010).

32. H. Shi and Y. Liu, Journal of Jianghan Petroleum Institute, 25, 865 (2012).

33. D. Wu and J. Cao, Optics and Precision Engineering, 21, 1598 (2013).

34. Y. Huang, K. B. Englehart, B. Hudgins and ADC. Chan, A
Gaussian mixture model based classification scheme for my-oelectric control of powered upper limb prostheses, IEEE Transactions on Biomedical Engineering, (2005), 1801-1811.

35. Q. Qiu, Appl. Comput. Math. 10, 340 (2011).

36. X. Yuan, Y. Wang and W. Sun, Computer Technology and Development, 12, 114 (2006).

37. T. Wang, F. Liu, M. Xiao and J. Chen, J. Comput. Theor. Nanosci. $10,742(2013)$.

38. R. E. Fan, P. H. Chen and C. J. Lin, Journal of Machine Learning Research, 6, 1889 (2005).

39. Burges, C.J.C., Data Mining and Knowledge Discovery, 121 (1998).

40. X. Liu, M. Zhu, Z. Chen and S. Chen, Computer Science, 30, 113 (2003).

41. Radu-Emil Precup, Tamás Haidegger, Stefan Preitl, Zoltán Benyó, Adrian Sebastian Paul and Levente Kovács, Appl. Comput. Math, 11, 378 (2012).

42. C.W. Hsu and C.J. Lin, A Comparison of Methods for Multiclass Support Vector Machines, IEEE Transactions on Neural Networks. (2002) 415-425 